\title{
Thank You to Our Peer Reviewers!
}

In this last issue of the year, we would like to acknowledge everyone who completed peer reviews for $J A D P R O$ in the past year. Our reviewers, who are from a wide variety of oncology specialties, dedicate their expertise and time to provide insightful feedback on submitted manuscripts.

We hope that during the year we have fully given you our thanks and made our appreciation to you clear, but would like to further acknowledge your contributions here.

If you would like to become a peer reviewer for $J A D P R O$, please don't hesitate to contact the editors at editor@advancedpractitioner.com for more information about how to get involved.

-From the Editors of $J A D P R O$

Paula Anastasia, RN, MN, AOCN ${ }^{\circledast}$

Kristi Baker, MSN, APRN, FNP-C

Roberta Boon, MSN, FNP-BC, RN, OCN ${ }^{\circledR}, \mathrm{RN}-\mathrm{BC}$

Tami Borneman, RN, MSN, CMS

Jeannine M. Brant, PhD, APRN, AOCN ${ }^{\circledR}$

Kevin Brigle, $\mathrm{PhD}$, ANP

Kelly Bugos, MS, ANP-C, RN

Kathleen Burns, AGACNP-BC, OCN ${ }^{\circledR}$

Carlin Callaway, DNP, MS, RN, ACNP-BC, ACNS-BC, AOCNP

Christopher J. Campen, PharmD, BCOP

Jill Cassaday, PharmD, BCOP, BCPS

Leah Clark, ARNP

Amanda Crick, MSN, ANP-BC, RN-BC

Marianne Davies, DNP, ACNP, AOCNP ${ }^{\circledR}$

Mary Elizabeth Davis, RN, MSN, AOCNS ${ }^{\circledR}$

Morgane Diven, PharmD, BCOP

Jessica Eno, MS, PA-C

Anecita Fadol, PhD, RN, FNP, FAANP

Andrea Fehl, MSN, CRNP

Nataya Francis, MS, ARNP, AOCNP ${ }^{\circledR}$

Alexis Geppner, MLS(ASCP), PA-C

Elizabeth Gilbert, MS, PA-C

Theresa Wicklin Gillespie, PhD, MA, RN

Jason Glass, ACNP-BC

Jennifer Glass, MS, PA-C

Amy Goodrich, MSN, CRNP-AC

Carolyn Grande, CRNP, AOCNP

Heather Hampel, MS, LGC

Tina Harris, AOCNP ${ }^{\circledR}$, FNP

Amy Henderson, ANP

Brianna Hoffner, MS, ANP-BC, AOCNP ${ }^{\circledR}$

Megan Holder, MSN, RN, NP-C, AOCNP ${ }^{\circledR}$

Joyce Jackowski, MS, FNP-BC, AOCNP ${ }^{\circledR}$

Kate D. Jeffers, PharmD, BCOP

Patricia Karwan, DNP, APRN-BC

Patrick Kiel, PharmD, BCPS, BCOP
Kate Kravits, MA, RN, HNB, LPC, NCC, ATR-BC

Bernadette Labriola, FNP-C, MSN, RN

Mary Jane LaRoche, ANP-BC, AOCNS ${ }^{\circledR}$, ACHPN $^{\circledR}$

Colleen Lewis, NP

Sharon Lewis, DNP, APRN-NP, AOCNP ${ }^{\circledR}$

Monica Macik, PharmD, BCOP, BCPS

Kelley D. Mayden, MSN, FNP, AOCNP ${ }^{\circledR}$

Gretchen McNally, PhD, ANP-BC

Laura Melton, PhD, ABPP

Lynora Metoyer, DNP, FNP

Rita Musanti, PhD, APN-BC

Joyce Neumann, MSN, AOCN ${ }^{\circledR}$

Rupal O'Quinn, MD, FACC

Claire Pace, MSN, APRN

Patricia Palmer, RN, MS, AOCNS ${ }^{\circledR}$

Mary E. Peterson, MS, APRN, AOCNP ${ }^{\circledR}$

Julie Ponto, PhD, RN, ACNS-BC, AOCNS ${ }^{\circledR}$

Allyson Price, MPAS, PA-C

Anishia Rawther-Karedath, MS, RN, AGNP-BC

Jean Ridgeway, DNP, APN, NP-C, AOCN ${ }^{\circledR}$

Barbara Rogers, CRNP, MN, AOCN ${ }^{\circledR}, A N P-B C$

Kathy Sharp, MSN, FNP-BC, AOCNP ${ }^{\circledR}, C C D$

Gary Shelton, DNP, NP, ANP-BC, AOCNP ${ }^{\circledR}, \mathrm{ACHPN}^{\circledR}$

Mady Stovall, RN, MSN, ANP-BC

Virginia Sun, PhD, RN

Rashida Taher, MPH, PA-C

Gregory Tallman, PharmD, MS, BCPS

Susan Tavernier, PhD, RN, APRN-CNS, AOCN ${ }^{\circledR}$

Katelyn Toeniskoetter, PharmD

Laura Trissel, NP

Wendy $\mathrm{H}$. Vogel, MSN, FNP, AOCNP ${ }^{\circledR}$

Elizabeth Waxman, RN, MSN, AOCN ${ }^{\circledR}, N P-C$

Jennifer Webster, MN, MPH, RN, AOCNS ${ }^{\circledR}$

Rita Wickham, PhD, RN, AOCN ${ }^{\circledR}$

Debra Winkeljohn, RN, MSN, AOCN ${ }^{\circledR}$, CNS

Laura Zitella, MS, RN, ACNP-BC, AOCN ${ }^{\circledR}$ 